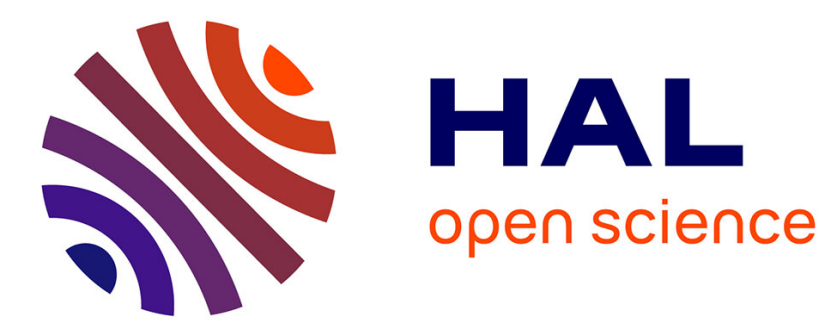

\title{
Indexicaux et parenthèses : articulation du discours et reprise en boucle \\ Francis Cornish
}

\section{To cite this version:}

Francis Cornish. Indexicaux et parenthèses: articulation du discours et reprise en boucle. Connexion et indexation. Ces liens qui tissent le texte, ENS Éditions, 2016, 9782847887983. 10.4000/books.enseditions.6847 . hal-02017869

\section{HAL Id: hal-02017869 \\ https://hal-univ-tlse2.archives-ouvertes.fr/hal-02017869}

Submitted on 13 Feb 2019

HAL is a multi-disciplinary open access archive for the deposit and dissemination of scientific research documents, whether they are published or not. The documents may come from teaching and research institutions in France or abroad, or from public or private research centers.
L'archive ouverte pluridisciplinaire HAL, est destinée au dépôt et à la diffusion de documents scientifiques de niveau recherche, publiés ou non, émanant des établissements d'enseignement et de recherche français ou étrangers, des laboratoires publics ou privés. 


\section{Indexicaux et parenthèses : articulation du discours et reprise en boucle ${ }^{1}$}

(Chapitre publié dans Connexion et indexation. Ces liens qui tissent le texte, recueil dirigé par L. Sarda, D. Vigier et B. Combettes. ENS Éditions, Lyon, 2016, pp. 137-154)

Francis Cornish

Université de Toulouse-Jean Jaurès et CLLE-ERSS, CNRS UMR 5263

\section{Introduction}

Le discours revêt une structure hiérarchique, formée de segments qui assument une relation de premier, de moyen ou d'arrière-plan par rapport à d'autres segments qui co-apparaissent avec eux (voir Khalil 2005, Pu 2011, Cornish 2012). Ces segments peuvent également être reliés entre eux d'une manière paratactique, comme unités «sœurs » qui partagent la même relation d'ancrage («grounding ») par rapport à un autre segment qui les domine au plan hiérarchique (voir Walker 1998 sur ce point). Et le fonctionnement des indexicaux (anaphoriques, «anadéictiques » et déictiques) s'avère tributaire, entre autres facteurs, du statut hiérarchique des unités de discours en jeu, ainsi que de leur relation (Pu 2011, Cornish 2011 et 2012).

Les digressions ou « insertions parenthétiques » qui souvent (surtout dans les échanges interactives à l'oral, mais aussi à l'écrit) interrompent un segment de discours, constituentelles des unités de discours à part entière, ou ne sont-elles que de simples interruptions sans effet sur la structure hiérarchique du discours ?² Cette contribution tente de répondre à cette question, en examinant surtout le comportement des indexicaux du segment suivant la digression, qui pointent vers une entité évoquée dans un segment pré-parenthétique. La nature de l'expression indexicale choisie pour reprendre un référent évoqué avant la digression peut donc, en principe, révéler la présence entre deux énoncés (ou deux fragments d'un même énoncé) d'une simple interruption sans effet sur la structure hiérarchique du discours en gestation, ou bien l'existence d'une véritable unité d'arrière-plan à part entière.

\section{Préliminaires analytiques}

\subsection{La distinction tripartite entre texte, contexte et discours}

Établissons d'emblée un certain nombre de distinctions terminologiques et conceptuelles, qui nous seront utiles pour l'étude à venir. D'abord, la triple distinction entre les dimensions texte, contexte et discours.

Dans la littérature sur l'analyse du discours, on confond souvent les termes de texte et de discours. Pour nous, le texte peut être envisagé comme la trace d'au moins un acte d'énonciation, produit sous une forme soit verbale, soit non-verbale (soit bien entendu les deux à la fois, en interaction l'une avec l'autre). Le texte revêt un caractère linéaire, à la différence du discours qui, lui, consiste en l'interprétation en ligne, continue et sujette à révision qu'effectuent les interlocuteurs de l'événement communicatif en déroulement. ${ }^{3}$ Cette

\footnotetext{
1 À paraître dans Sarda, L., Vigier, D. et Combettes, B. (dir.), Connexion et indexation. Ces liens qui tissent le texte (ENS Éditions, Lyon, mai 2016).

${ }^{2}$ Quoi qu'il en soit, les «parenthèses » sont par définition des unités d'arrière-plan, au niveau du discours.

${ }^{3}$ Ces propriétés différentielles vont s'avérer fort pertinentes dans notre étude des «insertions parenthétiques 》 (voir les sections 3 et 4 ci-dessous), car bien que celles-ci s'intercalent entre les segments primaires sur le plan textuel, leurs interprétation et fonction discursives se superposent à la représentation mentale des segments textuels primaires interrompus,
} 
interprétation est convertie par la suite en une représentation mentale, capable d'être hébergée dans la mémoire à long terme.

Les unités discursives (qui ne correspondent pas nécessairement à des constituants syntaxiques, sur le plan textuel) sont structurées, d'une part en unités d'arrière-plan, qui servent à ancrer les représentations discursives centrales à la fois modalement et référentiellement ; et d'autre part en unités de premier plan, qui, elles, sont susceptibles de faire progresser la communication. La structuration du discours en plans hiérarchiques permet la réalisation de ces différentes fonctions. Le discours est redevable donc en même temps au texte et au contexte. En revanche, la trace textuelle d'une suite connexe d'énonciations est transitoire, car une fois le discours construit, elle ne peut guère être maintenue en mémoire à court terme et encore moins à long terme - du moins, dans l'emploi normal, habituel de la langue.

Pour ce qui concerne le contexte, il revêt plusieurs aspects, dont le plus important est certainement la situation d'énonciation: celle-ci fonctionne comme point d'ancrage par défaut pour le discours à construire. D'autres aspects sont les relations interlocutives toujours en évolution, le domaine de discours à l'œuvre, le cadre socio-culturel de l'échange, le cotexte, le discours déjà construit et le genre de discours supposé. Le contexte de chaque énoncé est créé ou recréé de manière continue tout au long de la communication. ${ }^{4}$

Or, la triple distinction qu'on vient de voir est cruciale pour une caractérisation du fonctionnement de la référence indexicale (deixis, anaphore et « anadeixis » - voir plus bas): le texte ne fournit à cet égard que des indices perceptibles - le déclencheur d'antécédent et le marqueur indexical, dans leur co-texte respectif - ; mais c'est le discours, lui, qui rend disponible et accessible (grâce à une interaction complexe entre co-texte et contexte) une représentation mentale, évoluant au fil de la communication, de l'objet de discours ainsi ciblé. Voir Cornish $(2010,2011)$ pour une discussion plus ample de tous ces facteurs.

\subsection{Deixis, anaphore, "anadeixis », et les propriétés indexicales distinctives de divers types d'expressions liées au contexte}

La deixis et l'anaphore sont des procédures indexicales qui servent à gérer la coordination de l'attention des participants du discours. C'est par la construction, la modification et l'accession au contenu de modèles du discours en déroulement que l'utilisateur les exploite. Ces modèles sont représentés mentalement par chacun des participants au discours au fur et à mesure du déroulement de l'événement communicatif.

De façon prototypique, la deixis fonctionne pour orienter le foyer d'attention de l'allocutaire vers un nouvel objet de discours qu'il est invité à se représenter mentalement, par défaut à partir de la situation d'énonciation - dont le noyau (l' " origo » chez Bühler, 2009[1934]) est l'activité verbale et non-verbale du locuteur (et de l'allocutaire, devrait-on ajouter). La deixis permet ainsi d'ancrer le discours à construire en fonction du texte interprété dans un contexte pertinent : à ce titre, elle met en place à chaque fois un contexte nouveau en établissant les valeurs particulières des paramètres contextuels essentiels ${ }^{5}$ pour la communication.

L'anaphore (de discours), quant à elle, constitue une instruction tacite à conserver le foyer d'attention déjà en vigueur lors du dernier acte d'énonciation - autrement dit, l'état du modèle de discours pré-existant lors de l'emploi de l'anaphorique qui, au sein de sa

\footnotetext{
au plan du discours (voir aussi Forget 2000).

${ }^{4}$ Voir aussi Auer (2009) et Cornish (2009) à ce sujet.

${ }^{5}$ Ces paramètres sont l'espace déictique, le moment déictique, les rôles de locuteur actuel et d'allocutaire actuel assignés à l'un ou l'autre participant du discours, et la source du point de vue en jeu.
} 
prédication «hôte », réalise cette procédure référentielle discursive. Cette procédure est mise en œuvre grâce à l'emploi de marqueurs indexicaux peu proéminents phonologiquement (typiquement, les pronoms de $3^{\text {ème }}$ personne, les pronoms nuls, et les $\mathrm{SN}$ définis ou possessifs réduits).

Cependant, la relation entre deixis et anaphore n'est pas 'symétrique' : selon Lyons (1975) (cf. aussi Bühler 1934/2009, Gerner 2009 et d'autres), l'anaphore dépend de la deixis et est secondaire ainsi que dérivée par rapport à celle-ci (à la fois sur les plans ontogénétique et phylogénétique); car la deixis est une procédure référentielle plus élémentaire. La vraie relation entre ces deux procédures indexicales peut se représenter comme une solution de continuité, avec un moyen terme, qu'Ehlich (1982) a appelé « anadeixis ».

L' « anadeixis » réunit les procédures anaphorique et déictique dans des proportions différentes. Selon nous, il existerait au moins trois sous-types d' « anadeixis »:

- l'anadeixis 'stricte' : la référence ultérieure à un objet de discours qui aurait été évoqué en amont dans un discours, mais qui n'est plus - ou n'est pas encore- topical au moment où ce renvoi est effectué ;

- l'anadeixis de reconnaissance/de rappel : le pointage indexical vers un référent -qui peut être un événement, parfois stéréotypique- qui est supposé connu des interlocuteurs, donc représenté dans la partie commune de leur mémoire à long terme ; et

- la deixis de discours : le fait d'effectuer un « pointage cognitif » vers une représentation discursive accessible en mémoire de travail, et de créer par inférence une nouvelle entité discursive à partir de celle-ci. Cette entité sera ainsi pour partie nouvelle et pour partie ancienne au niveau du discours.

La Figure 1 présente sous forme d'une échelle les propriétés indexicales de 10 grandes catégories de marqueurs indexicaux. ${ }^{6}$

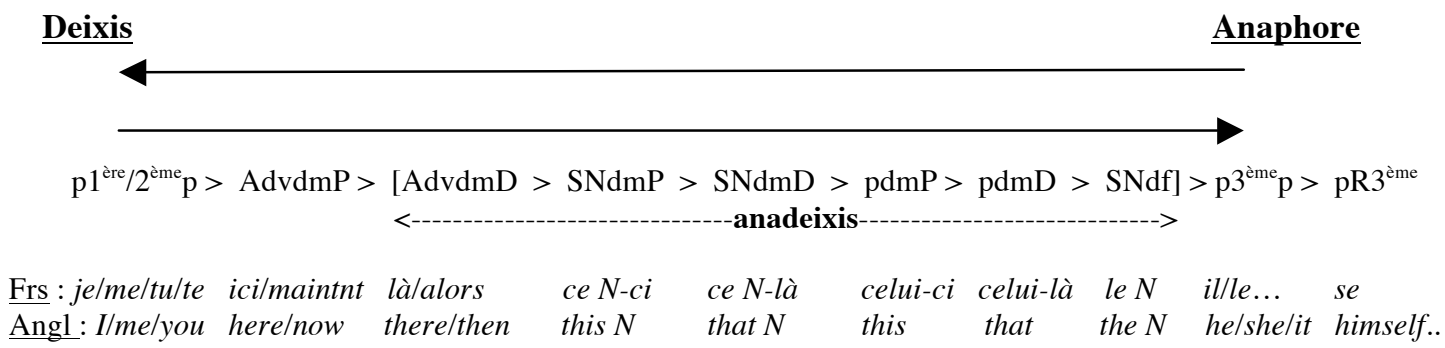

Figure 1: Echelle d'indexicalité encodée par certaines catégories d'expressions indexicales (Figure 1 dans Cornish, $2010: 116$ - révisée)

Cette échelle est fondée sur le degré d'indexicalité inhérent à chacune des catégories indexicales représentées. Le pôle 'Deixis' est occupé par les pronoms personnels de $1^{\text {ère }}$ et de $2^{2 \mathrm{ème}}$ personne : ces marqueurs sont intrinsèquement déictiques et fonctionnent sur le mode «token-reflexif». De ce fait, ils ne sont pas susceptibles d'un emploi anaphorique. Le pôle 'Anaphore', quant à lui, est représenté par les pronoms réfléchis (clitiques en français) de $3^{\text {ème }}$ personne : ce type d'expression ne peut fonctionner, dans son emploi prototypique, du moins, qu'au sein d'une proposition en termes de liage. Il s'agit là d'une fonction purement anaphorique ('stricte', donc). Son mode de fonctionnement est ainsi très contraint. Par ailleurs, la référence d'une occurrence de ces deux types de marqueurs «polaires » se met en place de manière quasi-automatique : le compreneur ne se voit pas contraint d'invoquer le

\footnotetext{
6 Voici la liste des abréviations qui sont utilisées dans la Figure 1 : ‘p $1^{\text {ère }} / 2^{\text {ème }} / 3^{\text {ème }}$ p': «pronom de première/deuxième/troisième personne»; 'P': «proximal»; ' $\mathrm{D}$ ': «distal»; 'dm': «démonstratif»; 'adv': «adverbe»; 'SN': «syntagme nominal»; 'p': «pronom»; 'df': «défini»; 'R': «réfléchi».
} 
contexte plus large du marqueur concerné afin de déterminer leur référent, comme cela est le cas avec les autres marqueurs sur l'échelle.

Pour ce qui concerne l'empan de l'Echelle dénommé « Anadeixis », la plupart des marqueurs concernés sont des types d'expressions à base démonstrative. Ces marqueurs démonstratifs (adverbes, SN et pronoms) se voient rangés en fonction de la distinction proximale (marquée) versus distale (non marquée) qu'à l'occasion ils manifestent sur le plan morphologique. Ainsi, la variante marquée revêtirait un degré plus grand de 'déicticité' que son homologue non marqué (cf. Langacker, 2002 : 34 ; Lyons, 1975). Nous avons placé les adverbes démonstratifs (maintenant/alors, ici/là en français, et now/then, here/there en anglais) à une position plus haute - donc plus à gauche sur l'échelle de la Figure 1- que les SN lexicaux ; et corrélativement, les SN lexicaux assument une position plus élevée que les pronoms correspondants. De même, toutes les catégories à base démonstrative se trouvent plus à gauche par rapport aux SN définis : ceux-ci ont été placés à la frontière droite du segment « Anadeixis » dans l'échelle, puisque sans être cantonnés à remplir uniquement une fonction indexicale, ${ }^{8}$ ils sont susceptibles de réaliser des fonctions soit déictiques, soit anadéictiques 'stricts', soit même anaphoriques. Voir Cornish (2010 et 2011) pour plus d'informations à ce sujet.

\section{Les caractéristiques essentielles des « parenthèses »}

Examinons à présent les aspects relevant du texte et du discours (cf. § 2.1) et la pertinence de cette distinction pour une caractérisation des parenthèses dans un discours: en effet, les parenthèses ou digressions par rapport à la ligne centrale du propos en développement dans un discours ont plusieurs propriétés relevant de l'un ou de l'autre de ces deux plans.

Selon Debaisieux et Martin (2010: 311), les véritables «parenthèses », espèces de digressions ou de décrochages par rapport au discours en cours d'élaboration au moment de l'insertion, « constituent (...) des énonciations indépendantes, des unités macro-syntaxiques qui ne sont reliées au contexte par aucune relation micro-syntaxique », même là où elles sont introduites par des subordonnants (par exemple, puisque ou parce que). Selon les auteurs, aucune propriété syntaxique inhérente aux parenthèses ne les caractérise en tant que telles. Au niveau de l'oral interactif, celles-ci ne sont pas intégrées prosodiquement à leur contexte gauche et/ou droit, à la différence des incises (par exemple comme s'ils y étaient ou si on peut dire ${ }^{9}{ }^{9}$

Pour Richard et Le Bot (2008 : 108), comme pour bien d'autres, « la parenthèse est un indice qui invite à prendre en compte un point de vue différent. C'est un système d'alerte qui indique que le locuteur change de palier énonciatif. » Les auteurs proposent (ibid.p. 106) une définition plus restrictive des parenthèses à l'oral par rapport à l'écrit (le terme est importé de l'écrit où il relève à strictement parler de la ponctuation, qui est propre à ce médium) : pour qu'il y ait parenthèse à l'oral, non seulement il faut qu'il y ait décrochage au niveau énonciatif par rapport à ce qui précède, mais le début et la fin de la séquence concernée doivent être marqués formellement (syntaxiquement, prosodiquement ou par des répétitions ou reformulations). A l'écrit, elles sont repérables selon des marques de ponctuation spécifiques (séquences contenues par des marques ouvrantes et fermantes: virgules, tirets, crochets ou

\footnotetext{
${ }^{7}$ Voir de Mulder et Vetters (2008) sur l'adverbe temporel maintenant, et Kleiber (2008) pour ici et là. Pour les premiers, l'adverbe proximal maintenant ne réalise que la deixis, et son homologue temporel distal, alors, fonctionne de préférence anaphoriquement. Pour Kleiber, il en est de même pour la paire d'adverbes spatiaux ici (proximal) et là (distal).

${ }^{8}$ En effet, il est tout à fait possible qu'ils réfèrent de manière autonome par leur seul contenu lexical, à condition que celui-ci soit suffisant pour permettre au compreneur de déterminer leur référent d'une manière non-équivoque.

${ }^{9}$ Voir Marandin (2008) pour une analyse approfondie des incises et incidentes en français. Voir Debaisieux (2008a) pour la distinction entre véritables parenthèses et incises.
} 
parenthèses «formelles »). Pour Forget (2000 : 16-17), en revanche, il est vain de chercher à circonscrire ce phénomène par des facteurs purement textuels, car il est foncièrement d'ordre discursif.

Pour Gachet (à paraitre : § 6.4), en l'absence d'indices grammaticaux, les parenthèses se caractérisent par des propriétés sémantiques plutôt que syntaxiques : «En dehors des cas particuliers où le statut grammatical fournit des indices (...), c'est l'évaluation de son contenu sémantique qui permet de repérer la parenthèse » (je souligne).

Un des apports significatifs de la contribution de Gachet est sa distinction entre deux types de "procédés» d'enchaînement que réaliseraient les locuteurs à l'aide des «parenthèses »: le procédé 1 , où le locuteur emploie, à la sortie de la parenthèse, un marquage signalant un enchaînement entre les segments qui l'encadrent, et le procédé 2, par lequel c'est le discours primaire suivant qui est signalé comme devant s'enchaîner avec la parenthèse elle-même. Cependant, ces deux procédés ne sont pas mutuellement exclusifs, car ils peuvent s'appliquer simultanément. C'est le cas notamment de l'exemple (2) plus bas.

Clark (1996), pour sa part, propose une distinction très utile entre deux «pistes » discursives opérant en simultané au fur et à mesure que la communication se déroule (un peu comme sur une bande magnétique) : à savoir, une piste primaire et une piste secondaire. Dans la première, les interlocuteurs négocieraient conjointement la «transaction officielle » ('official business'), le propos central de l'échange; alors que la seconde, elle, serait spécialisée dans le «métadiscours », servant à gérer le discours à l'œuvre. C'est ici que les interlocuteurs signaleraient les objectifs et les fonctions de l'échange en déroulement.

Un exemple (tiré de l'anglais) provient d'une discussion radiophonique sur la façon dont l'Église médiévale aurait exploité la notion du 'Diable' comme instrument de contrôle social (BBC Radio 4, «Start the Week», 22 avril 1996). L'animateur Melvyn Bragg (MB) commence par remettre en question la conception qu'il suppose chez son invité Peter Stanford (PS) de cet état de choses. ${ }^{10}$

(1) MB: yes - the INteresting thing is that - was it already coercive an' repressive - or did it actually call out something that's in people anyway - I mean the way you put it is that this is an authorit this is a Church behaving as the Church behaved in many many diff(e)rent ways - in great ways an' in wicked ways - but this is a Church behaving in a very authoriTArian way - in saying "you will

5. follow us - or we will er - we will - GET you and we've GOT the man to get you - he's called 'the Devil", - but - isn't there something else there - isn't it a rec(og)nition of what's part of human nature and it was a BRILLiant metaphor - just as Christianity is full of brilliant metaphors as to what human nature is about so - .hhh it's more positive in a way than [what you're saying] PS: [well - well] it wouldn't have worked would it - un un unless they were actually tapping into

10. something that people wanted to believe - and if you THINK about it $\mathrm{MB}$ : =so you're agreeing=

PS: =I'm agreeing - but if you THINK about the - concept of evil . . (Exemple (2) dans Cornish, 2006 : 229, 'Understanding spoken discourse'. In K. Brown (ed.), Encylopedia of Language and

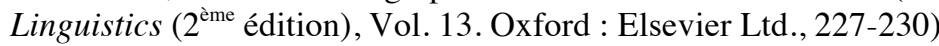

Cet exemple fournit une illustration nette de la structure à deux pistes du discours, selon Clark (1996). Le fait que la coordonnée finale du segment précédant l'interruption de la réponse de P. Stanford par Bragg (donc, à la ligne 10) ait été répétée par Stanford presque mot pour mot immédiatement après l'interruption en deux temps, indique que ce n'est pas la piste 1 , de « transaction officielle » du propos central, qui a été interrompue ; mais bien plutôt la piste 2, 'métadiscursive'. On pourrait interpréter comme s'inscrivant dans le cadre de la piste 2 de Clark ce que fait valoir Zay (1995 : 210) concernant la gestion « parallèle » par le locuteur des

\footnotetext{
10 Les symboles de notation employés ici (ainsi qu'ailleurs : voir l'exemple (5) plus bas) sont les suivants : - : pause; - : pause double ; lettres majuscules : syllabe fortement accentuée ; [ . .] : paroles simultanées ; = : suite de la part d'un interlocuteur sans interruption ; (a): élision de ' a' ; hhh : forte inspiration.
} 
énonciations principale et parenthétique, où figure un marqueur indexical qui n'aura sa pleine interprétation qu'en fonction de l'énonciation suivant la parenthèse.

\section{Les données...}

\section{1 ...françaises}

Voyons tout d'abord deux extraits oraux du corpus PFC (Phonologie du Français Contemporain), suivis d'un extrait écrit.

(2) 75 cabl Conversation guidée (corpus PFC)

[Sur les bombardements aériens pendant la seconde guerre mondiale en Bretagne]

...Mais euh, euh. Finalement euh quand euh nous avons été sous très gros bombardements, américains puis anglais et nous sommes (XX), après le bombardement anglais mon, mon père a dit 'non il f/ il faut partir il faut' tout le monde partait, lui il avait passé les quatre nuits, ou trois nuits, à, à ramper dans les décombres pour aider les gens à mourir, parce qu'on pouvait pas sortir les gens de là c'était impossible.

5. On s'est retrouvé sans eau, ni gaz, ni téléphone, ni électricité, et les pompiers, euh, euh c'était à (XX), la ville est assez haute, $<\mathrm{E} 1$ : Hum, hum.> et la, la Loire est en bas. ${ }^{11}$ Et les malheureux allaient puiser de l'eau dans la Loire et remontaient pour arroser voyez c'était, c'était effarant... (Cornish, $2010: 126$, exemple 7)

Dans cet extrait, l'interlocuteur ou le compreneur plus généralement identifie, rétrospectivement, 'les malheureux' dont il est question (ligne 6) avec 'les pompiers', qui viennent d'être mentionnés rapidement, « en passant» en somme, ligne 5-sans que la prédication dont ce SN allait être le sujet ne soit complétée. Cette introduction est suivie par une préparation par la locutrice de ce qui viendra après (telle est la fonction discursive de la parenthèse ici).

Ce segment effectue une parenthèse dans la narration, et motive ainsi le choix d'un indexical plus important qu'un simple pronom (le SN défini les malheureux ${ }^{12}$ comportant un « nom de qualité », qui traduit toute la subjectivité de ce renvoi), pour reprendre l'objet de discours en jeu. La dernière syllabe du nom tête de ce $\mathrm{SN}$ défini était prononcée avec une remontée de hauteur et une accentuation, ce qui pourrait signifier que la fonction discursive de ce marqueur ici n'est pas canoniquement «anaphorique », mais plutôt «anadéictique » (voir le $\S 2.2$ ci-dessus).

On pourrait analyser cet exemple comme étant « hybride », suivant la conception chez Gachet (à paraître) des phénomènes de sorties de parenthèses : car il relève en même temps du « procédé 1», puisque ici ce qui est prédiqué des 'pompiers' dans la proposition hôte s'y applique directement ; mais aussi du « procédé 2 », puisque le segment suivant la parenthèse s'enchaîne bien sur celle-ci. Car la conjonction et introduisant la reprise du discours coupé par la parenthèse est susceptible d'une lecture causale : 'C'est parce que la ville est assez haute et que la Loire est en bas que les « malheureux pompiers » devaient descendre jusqu'au fleuve puis remonter encore afin d'éteindre les incendies'. Il s'agit de ce que Debaisieux et Martin (2010 : 316) appellent un «cadrage par anticipation», où « le locuteur interrompt

\footnotetext{
${ }^{11}$ Les segments en gras dans les exemples présentés constituent les parenthèses.

12 Corblin (2005) met l'accent sur la relative rareté de ce type de marqueur à tête lexicale dans les « chaînes de conversation », qui renvoient à des entités censées être connues des participants au discours. Pour Corblin, en conversant, les interlocuteurs participent de ce qu'il appelle une «communauté épistémique » restreinte. Il en résulte que les «chaînes référentielles » et «anaphoriques » qui à l'oral sont constituées d'un nom propre repris par une suite de pronoms de $3^{\text {ème }}$ personne sont normales, attendues, à la différence d'une variation des marqueurs de reprise - par exemple, l'emploi de SN définis dont la tête lexicale varie. Ce dernier type de reprise est plus caractéristique, selon lui, de l'écrit-type de discours que Corblin appelle «à interlocuteur générique ».
} 
l'énumération ${ }^{13}$ et fournit à son interlocuteur des informations contextuelles lui permettant d'interpréter ce qui suit ».

Regardons à présent l'exemple (3), également tiré du corpus PFC :

(3) 11agm1 Conversation libre (corpus PFC)

(...) JP: Ca s'est fait, et ils ont acheté aussi le euh, le restaurant qu'il y a, au second feu rouge là. A Capendu. GM: Ah bon. JP: Oui pour faire, là, là, là il est bien placé d'ailleurs E: de commercialisation (peu clair). GM: ah, ils l'ont acheté? JP: Ils ont acheté GM: On le, on l'a jamais vu que ce soit à vendre. JP: Et bien hein c'est comme ça, oui il y a longtemps que c'est fermé hein.

5. GM: Oui, oui ça c'est mais tout, il travaillait hein. A/ ce restaurant, il travaillait quelque chose hein. JP: peut-être euh il est âgé alors, il était âgé ou je sais pas. GM: Non, c'était des jeunes hein. C'était les, comment il(s) s'appelai(en)t euh. Euh, [S2] je me rappelle pas son nom, on y faisait souvent les réunions R.P.R aussi, c'était un R.P.R le type (rires). On allait là manger. C. était venu et tout, même L. quand ils étaient présentés les, les députés, on était allé manger là au (énoncé suspendu) (...)

A strictement parler, le segment en gras ici ne constitue pas une «insertion parenthétique », car la construction en cours d'élaboration qu'elle interrompt n'est pas complétée (elle est abandonnée, comme c'est souvent le cas dans les conversations orales). Mais du point de vue énonciatif, il s'agit bien d'un décrochage "métadiscursif » par rapport à la ligne du discours primaire en développement avant l'amorce en question, et qui est reprise immédiatement après. Aux lignes 6-7, donc, GM amorce une construction attributive, ou même identificationnelle (C'était les...) puis oublie le nom de la ou des personne(s) qu'il était sur le point d'évoquer. Le segment en gras suivant cette amorce représente un décrochage énonciatif, une tentative du locuteur de retrouver ce nom, en essayant de relancer la mémoire commune de ses interlocuteurs par l'évocation des caractéristiques du ou des individu(s) concerné(s), afin de remémorer le nom manquant (on y faisait souvent les réunions R.P.R aussi, c'était un R.P.R le type). Cependant, aucun nom n'est proposé, et GM poursuit la discussion précédant l'interruption à propos du restaurant en question, en employant un marqueur démonstratif primaire (là), sur le mode « anadéictique 'strict'» (voir le $§ 2.2$ ), pour renvoyer au restaurant dont il était question auparavant et pour signaler la clôture de la parenthèse. ${ }^{14}$

Voici maintenant un exemple écrit :

(4) ITALIENS. Les plaines de la Garonne ressemblent à s'y méprendre à celles du Pô, c'est sans doute pourquoi plusieurs générations d'Italiens du Nord se sont si bien acclimatées. Originaires du Piémont, de Vénétie ou du Frioul, ils ont été nombreux au cours des trois grandes vagues d'immigration - la plus importante date des années $\mathbf{1 9 2 0}^{(\mathbf{1})}$ - à venir travailler la terre puis le

5. ciment. Les années 1950 à 1970 ont vu certains d'entre eux passer du métier de maçon au statut de promoteur immobilier. L'intégration s'est faite sans trop de difficultés - ceux qui ne parlaient pas français comprenaient le patois de Catinou et Jacouti sur Radio Toulouse ${ }^{(2)}-$ si bien qu'aujourd'hui on ne perçoit pas que cette «communauté » est la plus importante de notre région. M.F. (Extrait du Dictionnaire de Toulouse, dir. G. Santier, Portet-sur-Garonne : Nouvelles Editions Loubatières, 2004, p. 249)

Il y a deux parenthèses dans cet extrait (numérotées en exposant), délimitées par des tirets.

\footnotetext{
13 Une énumération est interrompue dans l'exemple analysé à ce point de l'échange par les auteurs. Plus généralement, il s'agit du discours primaire qui serait en développement au moment de l'interruption.

${ }^{14}$ A noter ici que le segment en gras dans la transcription de cet extrait était prononcé sur un registre plus bas que le texte environnant, et plus « doucement » également. Par contre, au début de la reprise du discours primaire, à la ligne 8 , la seconde syllabe du verbe allait était marquée par une remontée en hauteur et une accentuation, qui étaient restées élevées sur l'adverbe démonstratif là qui suit. Ces caractéristiques se retrouvent en anglais parlé également selon Dehé (2014:34-6), en ce qui concerne l'intonation et la prosodie des parenthèses au sein du texte « hôte ».
} 
Nous allons nous intéresser à la seconde uniquement. A l'évidence, cette digression sert discursivement à justifier l'affirmation dans la partie gauche du discours primaire, selon laquelle l'intégration des immigrés italiens aux alentours de Toulouse au cours du $20^{\text {ème }}$ siècle a été réussie: son rôle dans le discours en déroulement est à l'évidence de prévenir une objection que pourrait soulever le lecteur à l'affirmation qui vient d'être faite, à savoir que ceux parmi ce groupe d'immigrés italiens à l'époque qui ne parlaient pas français auraient bel et bien eu des difficultés à s'intégrer. Cependant, le SN démonstratif cette "communauté » dans le segment primaire post-parenthétique ne renvoie pas au sous-groupe d'immigrés italiens qui ne parlaient pas français, évoqué à l'intérieur de la parenthèse, mais fonctionne «en boucle» pour désigner l'ensemble des Italiens qui s'étaient installés dans la région. L'emploi du pronom personnel de $3^{\text {ème }}$ personne au pluriel $i l s,{ }^{15}$ lui, aurait renvoyé à ce sousgroupe, ne réalisant pas ainsi le programme discursif voulu par l'auteur.

Le fait que le scripteur ait entouré de guillemets le nom tête du SN cette communauté indiquerait qu'il le considère comme problématique (quelque peu inapproprié, vu le degré d'absorption de cette population dans la population existante) ; le concept qu'il véhicule ne peut donc pas être présupposé par le lecteur, d'où l'emploi d'un déterminant démonstratif et non de l'article défini pour l'introduire. Tout comme celui de l'adverbe démonstratif là de l'exemple (3), cet emploi peut être qualifié d' « anadéictique 'strict'».

\section{2 ...et anglaises}

Pour ce qui concerne les données anglaises, comme en $\S 4.1$, nous allons regarder d'abord deux extraits oraux, puis un écrit. Dans le cas de deux d'entre eux (les exemples (5) et (7)), la «parenthèse » concernée sépare deux énonciations complètes, et n'en interrompt pas une seule. Ce cas de figure est prévu par Debaisieux (2008a : 28, n. 2) et par Gachet et Avanzi (2008 : 59) entre autres, dans une acception large des insertions parenthétiques (l'exemple (2) plus haut en est un exemple français). Cependant à chaque fois, il s'agit bel et bien d'un décrochage énonciatif par rapport à la ligne centrale du discours en cours. Voici d'abord un court extrait d'un débat radiophonique sur les médias, et notamment la BBC à l'époque (2007). Il s'agit d'une intervention lors de l'émission Any Questions?, BBC Radio 4, 25 août 2007 :

(5) PV: I think there's a bit of a moral PANic around about er - about the BBC and it it's it's got several roots - I mean the Hutton inquiry ${ }^{16}$ er - ditched ([dI $\left.[\mathrm{t}]\right)$ the BBC in a in a fairly

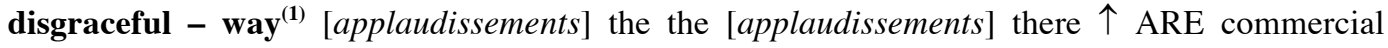
pressures on on on the $\mathrm{BBC}$ to to to to cut corners make programmes more cheaply - get ratings

5. which which produce these kind of - phone-in type er - programmes - whi- where all the scandals have been e e e even touching Blue Peter - erm trying to get $-\downarrow$ y'know $-\uparrow$ fake phone-ins $\downarrow$ and so forth $-\mathbf{e r}^{(2)}-$ but $\uparrow$ having SAID all that - erm I think that that the absolute CORE of the BBC is still a - a benchmark of of of of good journalistic - values it it it - the Corporation puts - puts money into er - documentaries which can take six months to - to to to make -

10. y'know not unlike newspapers which take six hours er to write er their er their pieces ${ }^{(3)}-$ er it it it's still got er the the OLD kind of CORE values - and there was an INteresting erm - er survey done - er today [accélère :] I'm not sure if it's been published yet] ${ }^{(4)}-$ but it it said that - seventy-three percent of the British public - TRUST the BBC - to be able to fix - its own problems - [downsteps :] an I thin(k) I'd go with the - the good common sense of the British public on this $\downarrow$ [applaudissements]

\footnotetext{
15 Ce pronom est pourtant employé en amont dans le texte, à la ligne 3.

16 «The Hutton inquiry » fait référence à l'enquête judiciaire menée par le juge Lord Hutton sur la mort de David Kelly, qui à l'époque avait recherché pour le compte des services secrets britanniques l'existence d'armes de destruction massive en Iraq (peu avant l'invasion de 2004). Son nom avait été rendu public par la BBC, alors qu'il tenait absolument à l'anonymat.
} 
Il y a plusieurs «parenthèses » ici (quatre en tout, numérotées en exposant). Examinons uniquement la troisième (lignes 8-10). Le début de cette séquence est surtout marqué par des hésitations et répétitions $\mathrm{du}$ pronom it, suivies d'une réparation indexicale via le remplacement de ce marqueur par le $\mathrm{SN}$ défini the Corporation (qui comme les pronoms est clairement anaphorique, de par sa réduction (ellipse de (the) British Broadcasting.... ${ }^{17}$ ) ; et la fin en est signalée par une reformulation du propos central esquissé avant l'interruption (er it it it's still got er the the OLD kind of CORE values). Cette séquence peut être interprétée comme fournissant une justification pour l'affirmation précédente selon laquelle la BBC serait « l'étalon d'or » des bonnes pratiques journalistiques, en la comparant favorablement à celles adoptées par la presse écrite. La continuation signalée par le pronom répété it du segment primaire pré-parenthétique indique la reprise du programme discursif central par le locuteur, sans que la parenthèse ne soit prise en compte ultérieurement (voir aussi à cet égard surtout l'exemple écrit (7) plus bas).

Voici à présent un autre exemple oral, tiré d'une conversation à propos des expériences d'une jeune Anglaise dans certaines villes de la côte ouest des États-Unis :

(6) $<$ x c8> i tended to stick to my drink oh apart from once when we found an irish bar in san francisco that was famous for its irish coffees (uh huh) and they had a long bar about fifty feet long with just rows and rows of glasses ready (uh huh) and as the girl came up for it was a singles bar i was being shown the singles bar ${ }^{(1)}$ and as the girl came up to shout your order one of the barmen there was about twenty of them behind the bar ${ }^{(2)}$ would rush up and sort of shovel so many glasses underneath the hot water tap and start doing the rigmarole for irish coffee it was very good irish coffee (uh huh) (Extrait de "The Great American Sandwich": conversation enregistrée faisant partie du projet de recherche HR5152, The Syntax of Scottish English (1977-1980), financé par le SSRC)

Il y a ici deux segments parenthétiques, toujours indiqués en gras. Dans le premier (lignes 34), la locutrice explique que le bar dont il est question était réservé aux célibataires ( « singles »). On note immédiatement que la parenthèse ici est signalée. ${ }^{18}$ C'est certainement pour respecter cette contrainte tacite que la locutrice $<x \mathrm{c} 8>$ avait maintenu le SN lexical défini réduit the girl dans la transition post-parenthétique de sortie, au lieu d'employer un marqueur plus atténué (un pronom de $3^{\text {ème }}$ personne au féminin). Comme la serveuse en question est conçue ici uniquement en tant que « rôle » dans un cadre particulier, elle occupe de ce fait une position purement d'arrière-plan. Ces facteurs pourraient nous amener à considérer cette parenthèse comme n'ayant aucune incidence sur la structure de discours à l'œuvre ici.

Considérons pour terminer l'interprétation du pronom de $3^{\text {ème }}$ personne dans la dernière phrase de l'extrait écrit suivant :

(7) (...) Enver [le père de Tracey Emin] went bankrupt and left when she was seven, and she squatted with her mother Pam and twin Paul in a staff cottage. "Mum crept into the hotel to steal lead piping to sell so we could eat." She disliked school and was not happy - a useful incubator for creativity... (Extrait de «If people say the bed's a joke, I'd say they're not interested in art »: entretien de l'artiste Tracey Emin par Andrew Duncan, Radio Times 1824/08/01, p. 32)

Ici, la parenthèse sert simplement à illustrer (donc à justifier l'affirmation du journaliste avant la parenthèse) l'extrême pauvreté dans laquelle la famille de Tracey Emin vivait quand

\footnotetext{
${ }^{17}$ En fait, nous pouvons dire ici que l'emploi de ce SN défini, dont la tête fait partie d'un nom propre, à la place du pronom ordinaire it, est en phase avec le « décrochage » discursif associé à la parenthèse - une rupture dans la continuité du discours primaire.

${ }^{18}$ Voir la contrainte postulée pour les parenthèses à l'oral par Richard et Le Bot (2008: 106), selon laquelle le début et la fin de celles-ci doivent être marqués formellement — ici par des répétitions : «...and as the girl came up...». Voir aussi l'exemple anglais présenté en (1) à cet égard.
} 
elle avait sept ans (le fait que sa mère s'est sentie obligée de voler des tuyaux de plomb pour les vendre afin d'acheter de quoi nourrir sa famille). Il s'agit d'une citation au discours direct, donc la source locutionnaire est le sujet de l'entretien, Tracey Emin, et non le journaliste auteur de l'article (Andrew Duncan). Le pronom féminin de $3^{\text {ème }}$ personne she sujet de la phrase suivant la parenthèse cible sans ambiguïté le sujet de l'entretien, Tracey Emin, l'entité macro-topicale de ce texte. Il ne peut renvoyer à l'individu féminin (la mère de TE), évoqué à l'intérieur de la parenthèse.

Cet état de choses peut s'expliquer par le fait que le segment par-dessus lequel le renvoi a eu lieu est précisément une citation au discours direct, attribuée au sujet de l'entretien : les guillemets entourant ce segment, ainsi que le passage de la $3^{\text {ème }}$ à la $1^{\text {ère }}$ personne au niveau des pronoms le signalent sans ambiguïté comme tel. Il est de ce fait clairement différencié de la ligne centrale du discours. Ainsi, le texte qui le suit continue le propos central, qui porte sur la suite de l'enfance de l'artiste TE : d'où l'emploi naturel d'un pronom de $3^{\text {ème }}$ personne dans ce sens, plutôt que d'un marqueur plus élaboré. Ce référent est donc toujours 'actif', en termes de foyer d'attention discursive, et la parenthèse est sans effet dans la structuration du discours à l'œuvre. Cette interprétation est aussi confirmée par la partie prédicative du segment postparenthétique : «disliked school and was not happy», propriétés qui ne pourraient guère se voir attribuées à la mère de TE.$^{19}$

\section{Conclusions}

Nos exemples montrent qu'on est en présence, selon le cas, soit de deux, soit de trois grands cas de figure, selon que la procédure indexicale exploitée pour la reprise est de type 'anadeixis stricte' ou 'anaphore canonique', et que le marqueur post-parenthétique employé est un SN lexical défini, une expression à base démonstrative ou un élément pronominal - et ce dans les deux langues représentées ici.

D'abord, quand le référent pressenti comme topical visé par un marqueur dans le texte central suivant la parenthèse n'est pas complètement installé (en tant que topique local du discours) avant l'occurrence de la parenthèse, alors ce marqueur peut prendre la forme d'un SN défini, à fonction anadéictique stricte. C'est le cas du SN défini les malheureux dans (2), de par la proéminence prosodique de sa prononciation ; cependant, the girl dans (6) est un cas à part, car ici le référent n'a pas une vocation topicale : son expression par un $\mathrm{SN}$ défini réduit relèverait du fait qu'il s'agit d'une entité stéréotypiquement attendue, ainsi que du besoin de démarquer la parenthèse (la répétition de l'expression utilisée juste avant la parenthèse). Dans les deux cas, l'élément lexical de ces SN sert surtout à caractériser ou à classifier (sur le mode de la présupposition) le référent visé - subjectivement dans le premier cas, objectivement dans le second-, en partie en fonction du contenu de la parenthèse elle-même. Dans le cas de (2), la parenthèse assume de ce fait une certaine importance dans la suite du discours. Dans ce dernier exemple également, l'emploi d'un pronom de $3^{\text {ème }}$ personne (ils) n'aurait pas convenu.

Dans le cas des exemples (3) et (4), le marqueur post-parenthétique utilisé indique clairement que la «parenthèse » est bien prise en compte par l'énonciateur dans la suite de son programme discursif. Dans chacun de ces cas, le marqueur choisi est en emploi

\footnotetext{
${ }^{19}$ Voir aussi l'analyse de l'extrait (11) par Ming-Ming Pu (2011, p. 53-54), extrait tiré d'une nouvelle anglaise, et son commentaire, où le macro-topique évoqué dans un segment de premier plan est repris même par un sujet zéro après l'intercalation d'un bref segment d'arrière-plan. Comme le soutient Pu (p. 54) à cet égard (je traduis), «La seconde occurrence d'un anaphorique zéro dans (11d) démontre bien comment le locuteur/scripteur emploie parfois l'anaphore (ainsi que la syntaxe) pour manipuler les représentations mentales du discours de l'auditeur/du lecteur en induisant ce dernier à négliger la rupture hors-ligne ( «off-line») et à focaliser son attention sur la ligne centrale du récit de même que sur le référent topical dans (11).»
} 
anadéictique strict, et non anaphorique, au sens propre du terme (voir le $§ 2.2$ supra). Dans (3) comme dans (4) (seconde parenthèse), des expressions démonstratives sont employées pour cibler des référents évoqués dans le texte central précédant l'occurrence de la parenthèse.

Par contre, dans (7), l'emploi d'un pronom de $3^{\text {ème }}$ personne sujet (she) dans le texte suivant la parenthèse est parfaitement naturel pour cibler en continuité, de façon nonambiguë, le référent macro-topical 'Tracey Emin' évoqué dans le texte central précédant cette parenthèse (citation au discours direct). Celle-ci relèverait donc de ce que Clark (1996) appelle la «piste 2 ». L'exemple (5) (la $3^{\text {ème }}$ parenthèse) est à l'évidence analogue à (7), car un pronom (répété plusieurs fois, à savoir it) est employé dans le texte central suivant la parenthèse pour renvoyer à une entité ('la BBC') évoquée avant celle-ci : la continuité anaphorique à l'état pur, donc.

En somme, le choix et l'emploi «en boucle» des marqueurs indexicaux postparenthétiques dans ces extraits reflètent la position de chacun d'entre eux sur l'Échelle de la Figure 1 -bien que la fonction métadiscursive des parenthèses à l'œuvre et leur contribution à la structuration du discours en cours (qui sont pourtant avérées), n'expliquent pas tout dans le choix de chaque marqueur. En outre, il ne s'agit pas uniquement d'identifier le référent visé par tel ou tel marqueur indexical : car en liaison avec leur prédication hôte dans son ensemble, leur fonction première est, à l'évidence, de structurer le discours à créer, en intégrant les unités déjà construites et à construire de telle ou telle manière.

\section{Remerciements}

Je voudrais remercier Frédéric Gachet, Laure Anne Johnsen, Jeanne-Marie Debaisieux et Béatrice Akissi Boutin de leurs remarques sur des versions antérieures de ce texte, ainsi que le correcteur d'ENS Éditions pour son aide précieuse concernant la forme du texte. Une présentation orale a été faite lors du colloque «Variations linguistiques », à 1'Université Mohammed 1 ${ }^{\text {er }}$, Oujda, Maroc, les 27-28 avril 2012.

\section{Références bibliographiques}

Auer, P. (2009). Context and contextualisation. In Verschueren, J. \& Östman, J-O. (eds.), Key Notions for Pragmatics. Amsterdam/Philadelphia: John Benjamins, 86-101.

Bühler, K. (2009)[1934]. Théorie du langage. La fonction représentationnelle du langage, Traduction, notes et glossaire par Didier Samain. Agone : Marseille. Collection Banc d'Essais.

Clark, H.H. (1996). Using Language . Cambridge : Cambridge University Press.

Corblin, F. (2005). Les chaînes de la conversation et les autres. In Gouvard, J-M. (dir.), De la langue au style. Lyon : Presses Universitaires de Lyon, 233-254.

Cornish, F. (2009). Text and discourse as context: Discourse anaphora and the FDG Contextual component. In Keizer, E. \& Wanders, G., (eds.), numéro thématique des Working Papers in Functional Discourse Grammar (WP-FDG-82): The London Papers I, 97-115.

Cornish, F. (2010). Indexicaux, discours, et mémoire discursive. Ce que les premiers révèlent du second et de la troisième. LINX 62-63 : 111-133.

Cornish, F. (2011). 'Strict' anadeixis, discourse deixis and text structuring. Language Sciences 33 (5) : 753-767.

Cornish, F. (2012). Micro-syntax, macro-syntax, foregrounding and backgrounding in discourse: When indexicals target discursively subsidiary information. Journal of Belgian Linguistics $26: 6-34$.

Debaisieux, J-M. (2008a). Les insertions de constructions à verbe fini dans la chaîne parlée : 
structures syntaxiques et modes de réalisation. Verbum XXX (1) : 25-35.

Debaisieux, J-M. (2008b). Énoncés parenthétiques et progression thématique : quelques observations sur corpus oraux. In Bertrand, O., Prévost, S., Charolles, M., François, J. et Schnedecker, C. (éds.), Discours, diachronie, stylistique du français. Études en hommage à Bernard Combettes. Berne : Peter Lang, 93-106.

Debaisieux, J-M. et Martin, P. (2010). Les parenthèses : étude macro-syntaxique et prosodique sur corpus. In : Béguelin, M-J., Avanzi, M. et Cornimboeuf, G. (dir.), La parataxe: Structures, marquages et exploitations discursives, Tome 2. Bern : Peter Lang, 307-337.

Dehé, N. (2014). Parentheticals in Spoken English. The syntax-prosody relation. Cambridge : Cambridge University Press.

De Mulder, W. \& Vetters, C. (2008). Le sens fondamental de maintenant : un token-reflexive. Cahiers Chronos 20: 15-33.

Ehlich, K. (1982). Anaphora and deixis: Same, similar, or different? In Jarvella, R.J. \& Klein, W., (eds.), Speech, Place and Action. Studies in deixis and related topics, Chichester: Wiley, 315-338.

Forget, D. (2000). Les insertions parenthétiques. Revue québécoise de linguistique 28 (2) : 1528.

Gachet, F. (à paraître). Sorties de parenthèses. In Groupe de Fribourg (éd.), Grammaire de la période II. Les parenthèses.

Gachet, F. et Avanzi, M. (2008). La prosodie des parenthèses en français spontané. Verbum $\mathrm{XXX}(1): 53-84$.

Gerner, M. (2009). Deictic features of demonstratives: A typological survey with special reference to the Miao group. Canadian Journal of Linguistics/Revue canadienne de linguistique 54 (1): 43-90.

Khalil, E. N. (2005). Grounding: Between figure-ground and foregrounding-backgrounding. Annual Review of Cognitive Linguistics 3: 1-21.

Kleiber, G. (2008). Comment fonctionne ICI? Cahiers Chronos 20 : 113-145.

Langacker, R.W. (2002). Remarks on the English grounding systems. In: Brisard, F. (ed.), Grounding. The epistemic footing of deixis and reference. Berlin \& New York: Mouton de Gruyter, 29-38.

Lyons, J. (1975). Deixis as the source of reference. In: Keenan, E.L. (ed.), Formal Semantics of Natural Language. Cambridge: Cambridge University Press, 61-83.

Marandin, J-M. (2008). Grammaire de l'incidence. Manuscrit non publié, URA 1028, Université de Paris 7.

Pu, M-M. (2011). Discourse Anaphora: A cognitive-functional approach. Munich: LINCOM EUROPA.

Richard, E. \& Le Bot, M-C. (2008). Pour une définition (très) stricte de la parenthèse à l'oral. Verbum XXX (1) : 103-117.

Walker, M. A. (1998). Centering, anaphora resolution, and discourse structure. Ch. 19 in Walker, M.A., Joshi, A.K. \& Prince, E.F. (eds.), Centering Theory in Discourse. Oxford: Clarendon Press, 401-435.

Zay, F. (1995). Notes sur l'interprétation des expressions référentielles dans les parenthèses. TRANEL 23 : 203-223. 